\title{
Diagnostic value of the combination of TruScreen and Pap smear in screening cervical epithelial lesions: Does it add advantages over the Pap smear alone?
}

\author{
Tajossadat Allameh $^{1}$, Somayeh Khanjani ${ }^{2}$, Fereshteh Mohammadizadeh ${ }^{3^{*}}$, Elaheh Refaei ${ }^{4}$ \\ ${ }^{1}$ Department of Obstetrics and Gynecology, School of Medicine, Isfahan University of Medical Science, Isfahan, Iran \\ ${ }^{2}$ School of Medicine, Isfahan University of Medical Science, Isfahan, Iran \\ ${ }^{3}$ Department of Pathology, School of Medicine, Isfahan University of Medical Science, Isfahan, Iran \\ ${ }^{4}$ School of Medicine, Isfahan University of Medical Science, Isfahan, Iran \\ Email: t_allameh@med.mui.ac.ir, somayeh.khanjani@yahoo.com, ${ }^{*}$ mohammadizadeh@med.mui.ac.ir, refaei@yahoo.com
}

Received 17 February 2013; revised 19 March 2013; accepted 27 March 2013

Copyright (C) 2013 Tajossadat Allameh et al. This is an open access article distributed under the Creative Commons Attribution License, which permits unrestricted use, distribution, and reproduction in any medium, provided the original work is properly cited.

\section{ABSTRACT}

Introduction: Pap smear is a well known test in screening of epithelial cell abnormalities of the cervix. However, adding other screening methods to this test may increase the sensitivity and specificity of case finding. Current study has been designed to assess the sensitivity and specificity of the combination of TruScreen and Pap tests in comparison to Pap smear alone in women referred for annual screening. Methods: This case-control study was conducted in two groups of 66 and 73 women with epithelial cell abnormality and normal results on Pap smear, respectively. Both groups were subsequently tested with TruScreen and colposcopy. Positive finding in any of the three studies made the patient candidate for biopsy as the standard diagnostic test. SPSS software was used to analyze sensitivity, specificity, positive predictive value, and negative predictive value of TruScreen, Pap smear, colposcopy and the combination of TruScreen and Pap tests. Results: 105 out of 139 women underwent biopsy. Of these, 32 (30.5\%) had abnormal result in biopsy. Combination of True screen and Pap smear led to a sensitivity of $\mathbf{9 3 . 8 \%}$ and specificity of $79.5 \%$ which means an improvement in both parameters. False negative rate decreased to $6.3 \%$ but false positive rate increased to $82.2 \%$. Positive and negative predictive values of the combined tests were $33.3 \%$ and $86.7 \%$, respectively. Conclusion: Combination of TruScreen and Pap smear is associated with a significant improvement in both sensitivity and specificity for early screening of preneoplastic and neoplastic cervical epithelial le-

\footnotetext{
"Corresponding author.
}

sions.

Keywords: TruScreen; Pap Smear; Colposcopy; Biopsy; Cervical Epithelial Lesions

\section{INTRODUCTION}

Carcinoma of cervix is a major cause of cancer related death among women. More than 80 percent of deaths caused by this cancer occur in developing countries. The still high incidence of cervical carcinoma in these countries is due to the lack of regular screening programs [1]. Pap smear followed by colposcopy is the traditional case-finding method in this disease [2]. However, Pap smear has some limitations in terms of sensitivity and specificity. The rate of false negative results in Pap smear varies from 20 to 40 percent and the test results are profoundly influenced by human errors in preparation and evaluation of the smear [3]. It has been shown that $47 \%$ of patients with cervical carcinoma had several Pap smears with normal results in the past [4]. Colposcopic evaluation to examine macroscopic changes of the cervix is profoundly dependent on the amount of training, visual skills, and experience of the examiner. Although colposcopy has an acceptable sensitivity in distinguishing normal tissue from abnormal one, it is less powerful in making distinction between severe and mild epithelial cell abnormalities [5]. Biopsy as the gold standard test is also an invasive, painful and stressful procedure. To overcome these problems, a device capable of providing and processing images from the cervix and offering probable diagnosis has been designed. This device can even be used by a trained nurse. This may be considered an important advantage in developing countries where 
limited resources and experts are sometimes great problems. Moreover, computer and internet technology provide the possibility of transferring the information to other centers for evaluating and providing treatment [5]. This technology may potentially increase the rate of cervical lesion diagnosis $[3,6]$. TruScreen is a real time device using electrical and optical signals to classify cervical tissue with an expert system approach. An expert system is a computerized device programmed to mimic the diagnostic capability of human. The TruScreen incorporates a portable console connected to a probeshaped hand-piece. The distal tip of the hand-piece is covered with a 5-mm disposable sensor element to protect against cross-infection. The device uses a combination of biosensors including directly reflected light, back scattered light, and electrical decay curves. Tissue is illuminated at four discrete wavelengths in the visible and infrared regions of the spectrum. In addition, the system incorporates electrical measurements of decay curves where the rate of electrical decay is inversely proportional to the degree of abnormality of the cervix. The information is filtered, sampled, and processed by a microcomputer within a portable console to extract the parameters of greatest value for tissue discrimination. The device is able to offer two diagnoses (normal and abnormal).

Several studies have been conducted to evaluate this system. In one multicenter study conducted in UK, adding TruScreen to Pap smear increased the sensitivity of Pap smear in diagnosing high grade squamous intraepithelial lesions from 69 to 93 percent. This combination also improved the sensitivity of diagnosing low grade squamous intraepithelial lesions from 45 percent to 87 percent in comparison to Pap smear alone [4]. In another study, 17 percent of women with normal Pap smear and abnormal TruScreen results were found to have abnormal colposcopy. On the other hand, 25 out of 37 women with ASCUS report or unsatisfactory specimen on Pap smear had normal TruScreen test. This normal status was confirmed by colposcopy in $76 \%$ cases. $92 \%$ of abnormal TruScreen results were confirmed by colposcopy [7]. In another study conducted in UK women experienced less anxiety and pain in TruScreen test and 82 percent of them preferred True screen to Pap smear [3].

The current study has been designed to determine the diagnostic value of the combination of TruScreen and Pap tests compared to Pap smear alone.

\section{METHODS}

This case-control study has been conducted in Shahid Beheshti University Hospital in Isfahan, Iran. Two groups of 66 and 73 women enrolled in the study. The first group had epithelial cell abnormality and the second one had normal results on Pap smear. Criteria for entering the study included married women between 15 to 65 years old referred for annual Pap test and the absence of hysterectomy and radiotherapy history as well as the absence of the history of cervical biopsy, LEEP, cauterization, and freeze procedures in the past three months. Informed consent was taken from the participants. Patients with Pap smear in the past 6 weeks and pregnant women were excluded from the study. Pap smears were taken in the outpatient clinic of the center. Plastic spatula and endocervical brush were used for ectocervical and endocervical sampling, respectively. Prepared smears were fixed with spray fixative and sent to pathology lab where they were examined by one pathologist and reported based on the Bethesda System 2001. Epithelial cell abnormalities on Pap smear included atypical squamous cells (ASC-US and ASC-H), low grade SIL, high grade SIL, and squamous cell carcinoma for squamous cells and atypical glandular cells (AGC of NOS and favor neoplastic types), adenocarcinoma in situ and invasive adenocarcinoma for glandular cells. Pap test in both groups of abnormal and normal results was followed initially by TruScreen performed by a technician who was unaware of the Pap results. TruScreen reported the status of the cervix as either normal or abnormal. Following TruScreen test both groups underwent colposcopy conducted by a colposcopist unaware of the results of both Pap smear and True screen test.

Colposcopy was performed as follow: after inserting speculum, cervix was stained with $3 \%-5 \%$ acetic acid for at least 30 to 60 seconds and then examined by white light. Acetic acid has no effect on glycogen-producing mature epithelium, but agglutinates nuclear and cytoplasmic proteins of metaplastic and dysplastic cells and creates a white tissue.

TruScreen is a real time device using electrical and optical signals to classify cervical tissue with an expert system approach. An expert system is a computerized device programmed to mimic the diagnostic capability of human. The TruScreen incorporates a portable console connected to a probe-shaped handpiece. The distal tip of the hand-piece is covered with a 5-mm-diameter single use sensore element designed disposable to protect against cross-infection. The device uses a combination of biosensors including directly reflected light, back scattered light, and electrical decay curves. Tissue is illuminated at four discrete wavelengths in the visible and infrared regions of the spectrum. In addition the system incorporates electrical measurements of decay curves where the rate of electrical decay is inversely proportional to the degree of abnormality of the cervix. The information is filtered and processed by a microcomputer within a portable console to extract the parameters of greatest value for tissue discrimination. The device is 
able to offer two diagnoses (normal and abnormal).

Positive finding in any of the three studies made the patient candidate for biopsy as the standard diagnostic test. Biopsy results were classified as normal and abnormal. Abnormal results on biopsy included low grade SIL, high grade SIL, and squamous cell carcinoma for squamous cells and low grade dysplasia, high grade dysplasia (adenocarcinoma in situ) and invasive adenocarcinoma for glandular cells.

Following final data collection, sensitivity, specificity, positive predictive value, and negative predictive value of TruScreen, Pap smear, colposcopy and the combination of TruScreen and Pap tests were determined using SPSS software version 20 and statistical tests including chi-square, T-test and ROC analysis.

\section{RESULTS}

TruScreen result was normal in $70(50.4 \%)$ and abnormal in $69(49.6 \%)$ of the women. The sensitivity, specificity, false positive rate, false negative rate, positive predictive value and negative predictive value of TruScreen test in comparison to Pap smear were $40.9 \%, 42.5 \%, 57.5 \%$, $59.1 \%, 39.1 \%$ and $44.3 \%$, respectively. Chart 1 shows these results. The degree of agreement between Pap smear and TruScreen was found to be $41.7 \%$ which is insignificant according to KAPPA statistical test $(\mathrm{P}=$ 0.084). In other words, there is not an appropriate agreement between Pap smear and TruScreen tests in diagnosing cervical lesions. Diagnostic values of TruScreen in comparison to Pap smear are shown in Table 1.

Colposcopy was normal in 48 (34.5\%) and abnormal in $91(65.5 \%)$ women. The sensitivity, specificity, false positive rate, false negative rate, positive predictive value and negative predictive value of TruScreen test in comparison to colposcopy were $58.2 \%, 66.7 \%, 33.3 \%$, $41.8 \%, 76.8 \%$ and $45.7 \%$, respectively. Results have been shown in Chart 2. The degree of agreement between TruScreen and colposcopy was $23 \%$ which is insignificant according to KAPPA test $(\mathrm{P}=0.078)$. Diagnostic values of TruScreen test in comparison to colposcopy are shown in Table 2.

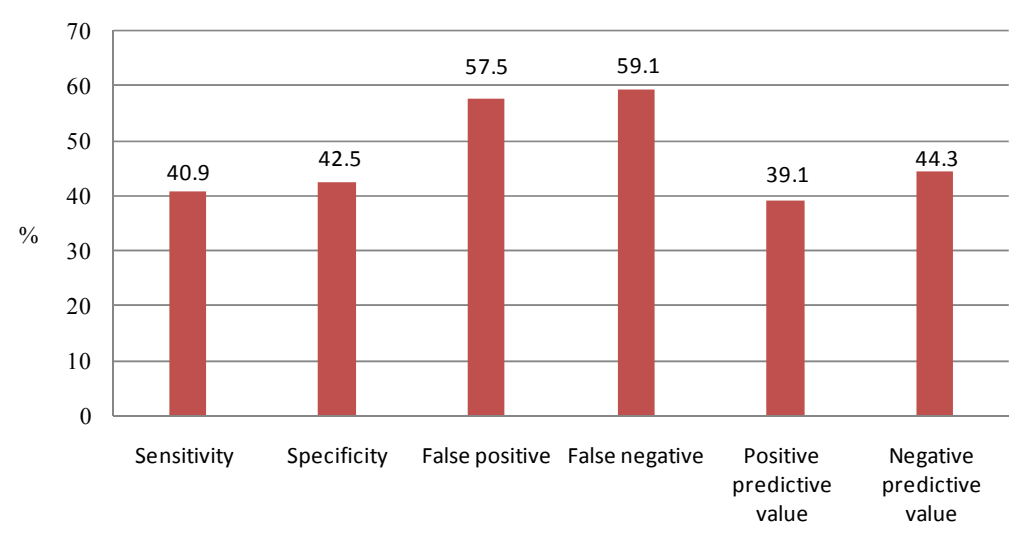

Chart 1. Diagnostic value of TruScreen test in comparison to Pap smear in diagnosing cervical lesions.

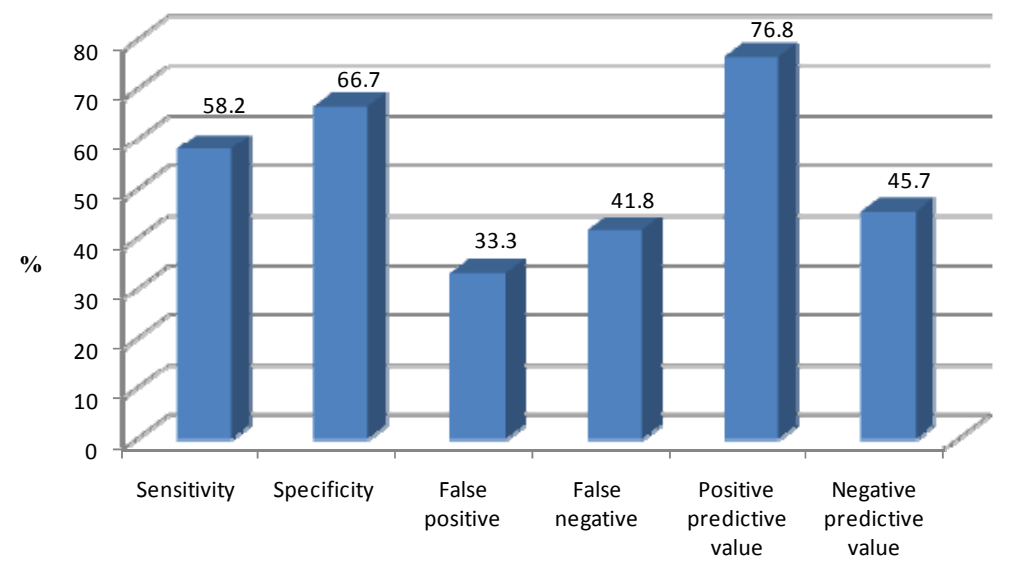

Chart 2. Diagnostic value of TruScreen test in comparison to colposcopy in diagnosing cervical lesions. 
Table 1. Frequencies of TruScreen results in terms of Pap smear.

\begin{tabular}{|c|c|c|c|c|c|c|}
\hline \multirow{2}{*}{$\begin{array}{l}\text { Pap smear } \\
\text { TruScreen }\end{array}$} & \multicolumn{2}{|c|}{ Normal } & \multicolumn{2}{|c|}{ Abnormal } & \multicolumn{2}{|c|}{ Total } \\
\hline & Number & Percent & Number & Percent & Number & Percent \\
\hline Normal & 31 & 42.5 & 39 & 59.1 & 70 & 50.4 \\
\hline Abnormal & 42 & 57.5 & 27 & 40.9 & 69 & 49.6 \\
\hline Total & 73 & 100 & 66 & 100 & 139 & 100 \\
\hline
\end{tabular}

Kappa agreement test $=41.7 ; \mathrm{P}=0.084$.

Table 2. Frequencies of TruScreen results based on colposcopy.

\begin{tabular}{ccccccc}
\hline \multirow{2}{*}{$\begin{array}{c}\text { Colposcopy } \\
\text { TruScreen }\end{array}$} & \multicolumn{2}{c}{ Normal } & \multicolumn{2}{c}{ Abnormal } & \multicolumn{2}{c}{ Total } \\
\cline { 2 - 7 } & Number & Percent & Number & Percent & Number & Percent \\
\cline { 2 - 7 } Normal & 32 & 66.7 & 38 & 41.8 & 70 & 50.4 \\
Abnormal & 16 & 33.3 & 53 & 58.2 & 69 & 49.6 \\
Total & 48 & 100 & 91 & 100 & 139 & 100 \\
\hline
\end{tabular}

Kappa agreement test $=0.23 ; \mathrm{P}=0.078$.

105 out of 139 women underwent biopsy. Biopsy results were normal and abnormal in $73(69.5 \%)$ and 32 $(30.5 \%)$ women, respectively. The sensitivity, specificity, false positive rate, false negative rate, positive predictive value and negative predictive value of TruScreen test in comparison to biopsy were $46.9 \%, 50.7 \%, 49.3 \%, 53.1 \%$, $29.4 \%$ and $68.5 \%$, respectively. Chart 3 shows these results. The degree of agreement between TruScreen and biopsy was $49.5 \%$ which is insignificant according to KAPPA test $(P=0.09)$. Diagnostic values of TruScreen test in comparison to biopsy are shown in Table 3.

Combination of TruScreen test and Pap smear led to sensitivity, specificity, false positive rate, false negative rate, positive predictive value and negative predictive value of $93.8 \%, 79.5 \%, 6.3 \%, 82.2 \%, 33.3 \%$ and $86.7 \%$, respectively. These results have been shown in Chart 4. Chart 5 presents diagnostic values of Pap smear, TruScreen, colposcopy and combined tests of TruScreen and Pap smear. Although combination of TruScreen test and Pap smear in comparison to Pap smear alone increases false positive rate, the combination decreases the false negative rate and improves sensitivity and specificity.

\section{DISCUSSION}

The main purpose of this study was to determine the diagnostic value of the combination of TruScreen and Pap smear in screening cervical epithelial lesions. Since cervical cancer is one of the most common cancers among women, attempts have been made to design cost-effec-
Table 3. Frequencies of TruScreen results in terms of biopsy.

\begin{tabular}{ccccccc}
\hline \multirow{2}{*}{$\begin{array}{c}\text { Biopsy } \\
\text { TruScreen }\end{array}$} & \multicolumn{2}{c}{ Normal } & \multicolumn{2}{c}{ Abnormal } & \multicolumn{2}{c}{ Total } \\
\cline { 2 - 7 } & Number & Percent & Number & Percent & Number & Percent \\
\cline { 2 - 7 } Normal & 37 & 50.7 & 17 & 53.1 & 54 & 51.4 \\
Abnormal & 36 & 49.3 & 15 & 46.9 & 51 & 48.6 \\
Total & 73 & 100 & 32 & 100 & 105 & 100 \\
\hline
\end{tabular}

Kappa agreement test $=0.49 .5 ; \mathrm{P}=0.09$.

tive devices capable of making distinction between normal and abnormal cervical epithelium. Pap smear, colposcopy and HPV DNA testing each have their own strengths and weaknesses. This study is based on the hypothesis that combination of an optoelectronic screening device and Pap smear increases the sensitivity and specificity of diagnosing cervical epithelial cell abnormalities. TruScreen is one of these devices with advantages of being cost-effective, noninvasive, safe and simple.

The results of study show that combination of TruScreen and Pap smear compared to Pap smear alone increases the sensitivity and specificity and reduces false negative rate of the screening program. However, this combination increases the rate of false positive results. Moreover, the combination has lower positive and negative predictive values compared to Pap smear alone.

Zaltkov study reports a higher sensitivity $(67 \%-70 \%)$ and lower specificity (81\%) for TruScreen in comparison to Pap smear and suggests the method suitable for independent primary screening and for adding the diagnostic assurance of the cytological screening [9].

The study of Singer et al. shows a significantly improved sensitivity for the combined TruScreen and Pap tests compared to the Pap smear alone [4]. This is the same as our finding about the sensitivity of the combined methods. Singer et al. suggest the adjunctive combination a useful method for very high detection rates of CIN [4].

Pruski et al. showed optoelectronic method as a useful screening tool for detection of neoplastic cervical changes [10].

According to our findings and those from other studies, it seems that the use of combination of TruScreen and Pap tests provides a high screening sensitivity and specificity. TruScreen provides a second opportunity to detect abnormality missed by cytology. The use of this combination provides a very high degree of assurance about the absence of a significant cervical epithelial cell abnormality when both tests yield negative results.

On the other hand, the significant increase in false positive rate of the combination in comparison to Pap smear alone leads to unnecessary additional evaluations 


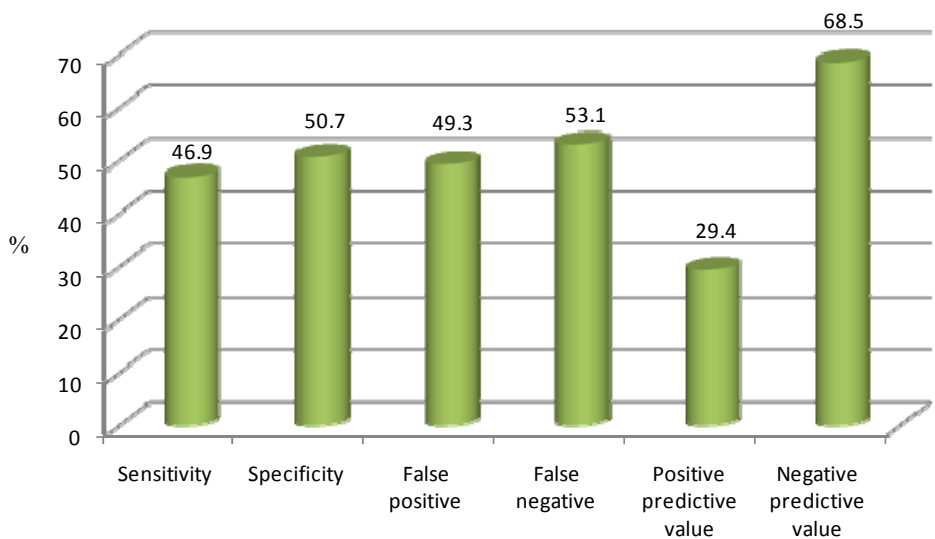

Chart 3. Diagnostic value of TruScreen test in comparison to biopsy in diagnosing cervical lesions.

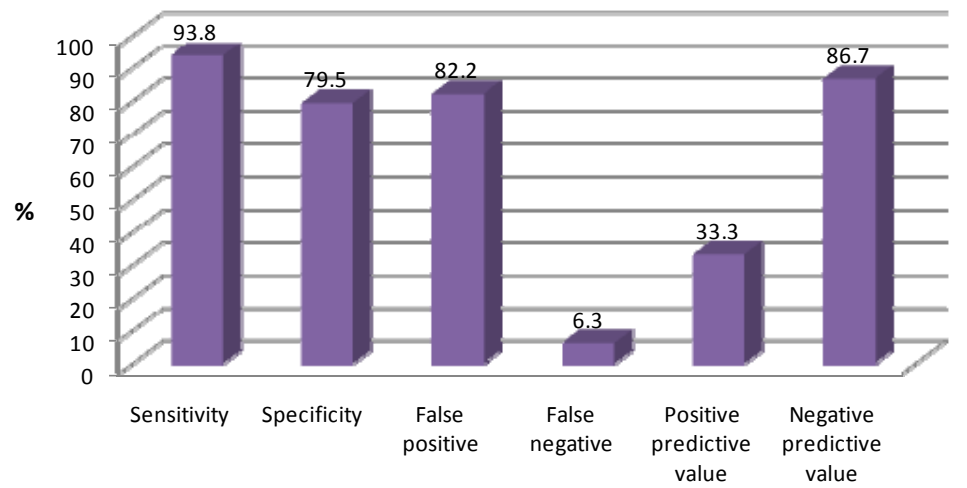

Chart 4. Diagnostic value of combination of TruScreen and Pap smear in comparison to biopsy in diagnosing cervical lesions.

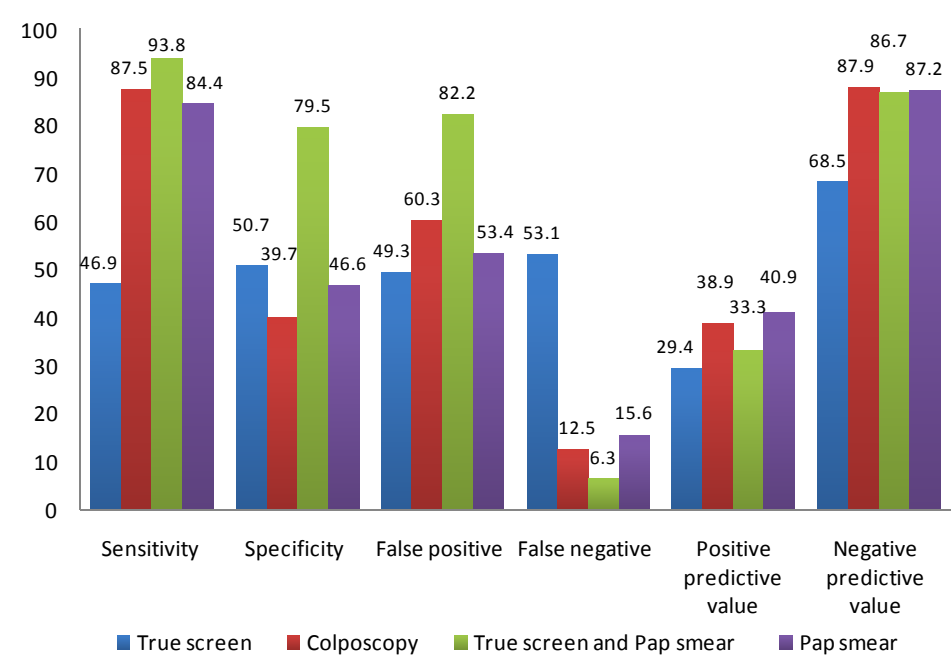

Chart 5. Comparing diagnostic values of Pap smear, TruScreen, colposcopy and the combination of Pap smear and TruScreen in diagnosing cervical lesions.

and imposes high economic costs on the health system. However, this burden would be negligible when the benefits resulted from higher sensitivity and specificity of the combined tests is considered. Since screening tests are expected to be available, easy to use, inexpensive and well-accepted by the patients, combination of these two 
methods seems reasonable in regions where more advanced diagnostic facilities are not easily available.

\section{REFERENCES}

[1] Mandelblatt, J.S., Lawrence, W.F., Gaffikin, L., Limpahayom, K.K., Lumbiganon, P., Warakamin, S., et al. (2002) Costs and benefits of different strategies to screen for cervical cancer in less-developed countries. Journal of National Cancer Institute, 94, 1469-1483. doi:10.1093/jnci/94.19.1469

[2] Nanda, K., McCrory, D.C., Myers, E.R., Bastian, L.A., Hasselblad, V., Hickey. J.D., et al. (2000) Accuracy of the Papanicolaou test in screening for and follow-up of cervical cytologic abnormalities: A systematic review. Annals of Internal Medicine, 132, 810-819. doi:10.7326/0003-4819-132-10-200005160-00009

[3] Quek, S.C., Mould, T., Canfell, K., Singer, A., Sklandnev, V. and Coppleson, M. (1998) The Polarprobe-Emerging technology for cervical cancer screening. Annals of the Academy of Medicine, Singapore, 27, 717-721.

[4] Singer, A., Coppleson, M., Canfell, K., Sklandnev, V., Mackellar, G., Pisal, N., et al. (2003) A real time optoelectronic device as an adjunct to the Pap smear for cervical screening: A multicenter evaluation. International Journal of Gynecological Cancer, 13, 804-811. doi:10.1111/j.1525-1438.2003.13393.x

[5] Louwers, J.A., Kocken, M., Ter Harmsel, W.A. and Verheijen, R.H. (2009) Digital colposcopy: Ready for use? An overview of literature. BJOG: An International Journal of Obstetrics \& Gynaecology, 116, 220-229. doi:10.1111/j.1471-0528.2008.02047.x

[6] Comppleson, M., Canfell, K. and Sklandnev, V. (2009) The Polarprobe-An instantaneous optoelectronic approach to cervical screening. CME Journal of Gynecologic Oncology, 31-38.

[7] Zanardi, C., Camerini, T. and Bucolo, C. (2001) TruScreen: A new ally in cervical cancer screening. Ginecorama, 26, 23-24.

[8] Addis, B.I., Hatch, K.D. and Berek, J.S. (2007) Intraepithelial diseases of cervix, vagina and vulva. In: Berek, J.S., Eds., Berek \& Novak's Gynecology, 14th Edition, Lippincott Williams \& Wilkins, Philadelphia, 561-600.

[9] Zlatkov, V. (2009) Possibilities of the TruScreen for screening of precancer and cancer of the uterine cervix. Akusherstvo i Ginekologiia (Sofiia), 48, 46-50.

[10] Pruski, D., Kedzia, W., Przybylski, M., Józefiak, A., Purol, M. and Spaczyński, M. (2010) Assessment of optoelectronic method and molecular test usefulness for cervical intraepithelial neoplasia and cervical cancer detection. Ginekologia Polska, 81, 426-430. 